\title{
Annealed Silver-Island Films for Applications in Metal-Enhanced Fluorescence: Interpretation in Terms of Radiating Plasmons
}

\author{
Kadir Aslan, ${ }^{1}$ Zoya Leonenko, ${ }^{2}$ Joseph R. Lakowicz, ${ }^{2}$ and Chris D. Geddes ${ }^{1,2,3}$
}

Received July 16, 2005; accepted July 29, 2005

\begin{abstract}
The effects of thermally annealed silver island films have been studied with regard to their potential applicability in applications of metal-enhanced fluorescence, an emerging tool in nano-biotechnology. Silver island films were thermally annealed between 75 and $250^{\circ} \mathrm{C}$ for several hours. As a function of both time and annealing temperature, the surface plasmon band at $\approx 420 \mathrm{~nm}$ both diminished and was blue shifted. These changes in plasmon resonance have been characterized using both absorption measurements, as well as topographically using Atomic Force Microscopy. Subsequently, the net changes in plasmon absorption are interpreted as the silver island films becoming spherical and growing in height, as well as an increased spacing between the particles. Interestingly, when the annealed surfaces are coated with a fluorescein-labeled protein, significant enhancements in fluorescence are osbserved, scaling with annealing temperature and time. These observations strongly support our recent hypothesis that the extent of metal-enhanced fluorescence is due to the ability of surface plasmons to radiate coupled fluorophore fluorescence. Given that the extinction spectrum of the silvered films is comprised of both an absorption and scattering component, and that these components are proportional to the diameter cubed and to the sixth power, respectively, then larger structures are expected to have a greater scattering contribution to their extinction spectrum and, therefore, more efficiently radiate coupled fluorophore emission. Subsequently, we have been able to correlate our increases in fluorescence emission with an increased particle size, providing strong experiment evidence for our recently reported metal-enhanced fluorescence, facilitated by radiating plasmons hypothesis.
\end{abstract}

KEY WORDS: Metal-enhanced fluorescence; radiative decay rate; increased excitation rate; radiative decay engineering; surface-enhanced fluorescence; silver island films; fluorescence spectroscopy.

\section{INTRODUCTION}

In the last five or so years our laboratories have both introduced and demonstrated many applications of

\footnotetext{
${ }^{1}$ Laboratory for Advanced Medical Plasmonics, Medical Biotechnology Center, Institute of Fluorescence, University of Maryland Biotechnology Institute, Baltimore, Maryland.

${ }^{2}$ Center for Fluorescence Spectroscopy, Department of Biochemistry and Molecular Biology, Medical Biotechnology Center, University of Maryland School of Medicine, Baltimore, Maryland.

${ }^{3}$ To whom correspondence should be addressed.E-mail: geddes@umbi. umd.edu.
}

metal-enhanced fluorescence [1-3]. These have included the increased detectability and photostability of fluorophores [4-6], improved DNA detection [7], the release of self-quenched fluorescence of overlabeled proteins [8], enhanced wavelength-ratiometric sensing [9], and the application of metallic surfaces to amplified assay detection $[1,10]$, to name but just a very few. In addition, we have developed many surfaces for metal-enhanced

Abbreviations: AFM, Atomic Force Microscopy; APS, 3-(aminopropyl)triethoxysilane; HSA, Human Serum Albumin; MEF, MetalEnhanced Fluorescence; RDE, Radiative Decay Engineering; SEF, Surface-Enhanced Fluorescence; SiFs, Silver Island Films. 
fluorescence [3,11-15], such as those comprised of silver islands [5,11], silver colloids [12], silver nanotriangles [13], silver nanorods [14], and even fractal-like silvered surfaces [15]. Several modes of silver deposition have also been developed, such as silver deposition by light [16] and electrochemically [17] on glass [5], plastics [18], and even electrodes [19].

In all of these applications of metal-enhanced fluorescence (MEF) to date, we have considered that the fluorophore is both excited and solely emits, the role of the surface plasmons being one of the near-field modification of the fluorophores' far-field spectral characteristics, i.e., a modification of the fluorophores' intrinsic radiative decay rate $[1-4,20]$. To this end we have found that the enhanced fluorescence signals (Quantum yields $-Q_{\mathrm{m}}$ ) of fluorophores in close proximity to metallic nanostructures could be described well by the following equations:

$$
Q_{\mathrm{m}}=\left(\Gamma+\Gamma_{\mathrm{m}}\right) /\left(\Gamma+\Gamma_{\mathrm{m}}+k_{\mathrm{nr}}\right)
$$

where $\Gamma$ is the unmodified radiative decay rate, $\Gamma_{\mathrm{m}}$ is the metal-modified radiative decay rate, and $\mathrm{k}_{\mathrm{nr}}$ are the nonradiative rates. Similarly, the metal-modified lifetime, $\tau_{m}$, of a fluorophore is decreased by an increased radiative decay rate:

$$
\tau_{\mathrm{m}}=1 /\left(\Gamma+\Gamma_{\mathrm{m}}+k_{\mathrm{nr}}\right)
$$

These equations have resulted in unusual predictions for fluorophore-metal combinations $[1-4,20]$. From Eqs. (1) and (2), we can see that as the value of $\Gamma_{\mathrm{m}}$ increases, the quantum yield $Q_{\mathrm{m}}$ increases, while the lifetime, $\tau_{\mathrm{m}}$, decreases. This is contrary to most observations in fluorescence [21], where the free-space quantum yield, $Q_{0}$, and lifetime, $\tau_{0}$, usually change in unison [21], as described by the well-known equations [21]:

$$
\begin{aligned}
Q_{0} & =\Gamma /\left(\Gamma+k_{\mathrm{nr}}\right) \\
t_{0} & =1 /\left(\Gamma+k_{\mathrm{nr}}\right)
\end{aligned}
$$

More recently, however, our interpretation of the same MEF results has shifted somewhat, to a model whereby nonradiative energy transfer occurs from excited distal fluorophores to the surface plasmon electrons in noncontinuous films, Fig. 1. The surface plasmons in turn, radiate (under certain conditions) the photophysical characteristics of the coupling fluorophores [22], in essence the system as a whole radiates. This subtle shift in interpretation has been facilitated by our recent findings of Surface Plasmon Coupled Emission (SPCE) [23-25], whereby fluorophores distal to a continuous metallic film can directionally radiate fluorophore emission at a unique angle from the back of the film. Remarkably, the plasmon-
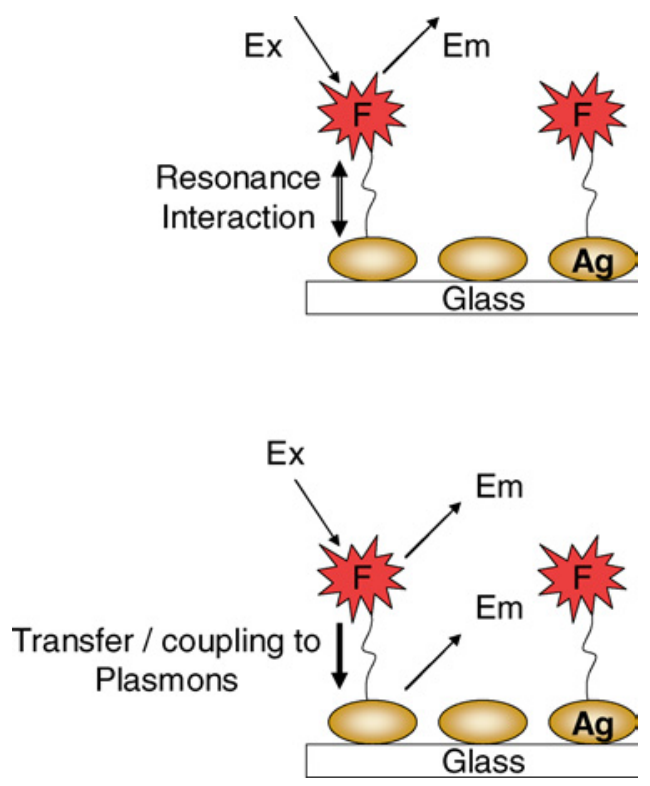

Fig. 1. Early interpretation of metal-enhanced fluorescence whereby fluorophores undergo a radiative decay rate modification-Top, and Bottom. Current thinking, the fluorescence emission is partially plasmon coupled and the system radiates.

coupled emission is nearly completely $p$-polarized, irrespective of the excitation polarization [23-25], strongly indicating that the emission is coupled through the surface plasmons.

Similarly, it has recently been hypothesized that metal-enhanced fluorescence is also plasmon coupled [22] and while not highly directional, as is the case with SPCE, is likely to be dependent on the plasmon scattering properties of the particulate nanostructures [22].

It is well-known that the extinction properties of metal particles can be expressed as both a combination of absorption $\left(C_{\mathrm{A}}\right)$ and scattering $\left(C_{\mathrm{S}}\right)$ factors, when the particles are spherical and have sizes comparable to the incident wavelength of light, i.e., in the Mie limit.

$$
C_{\mathrm{E}}=C_{\mathrm{A}}+C_{\mathrm{S}}=k_{1} \operatorname{Im}(\alpha)+\frac{k_{1}^{4}}{6 \pi}|\alpha|^{2}
$$

where $k_{1}=2 \pi n_{1} / \lambda_{0}$ is the wavevector of the incident light in medium I, $\alpha$ is the polarizability of the sphere with radius $r, n_{1}$ is the refractive index, and $\lambda_{0}$ the incident wavelength. The term $|\alpha|^{2}$ is square of the modulus of $\alpha$ [22].

$$
\alpha=4 \pi r^{3}\left(\varepsilon_{\mathrm{m}}-\varepsilon_{1}\right) /\left(\varepsilon_{\mathrm{m}}+2 \varepsilon_{1}\right)
$$

where $\varepsilon_{1}$ and $\varepsilon_{\mathrm{m}}$ are the dielectric and the complex dielectric constants of the metal, respectively. The first term 
represents the cross section due to absorption, $C_{\mathrm{A}}$, and the second term, the cross section due to scattering, $C_{\mathrm{S}}$. Current interpretation of metal-enhanced fluorescence is one therefore underpinned by the scattering component of the metal extinction, i.e., the ability of fluorophore-coupled plasmons to radiate [22].

In this paper we have studied the metal-enhanced fluorescence phenomenon from thermally annealed silver island films, SiFs. By annealing the surfaces we have been able to produce thermally stable $\mathrm{SiFs}$, which are not prone to further temperature effects. This suggests the re-use of these surfaces after autoclaving, i.e., a reusable generic surface for metal-enhanced fluorescence, or indeed, the use of these substrates for applications employing elevated temperatures, such as in MEF hybridization assays [7]. We have also chosen to anneal our freshly prepared silver island films in a conventional laboratory oven in the presence of oxygen. While this process is well-known to rapidly form oxide layers, particularly at high annealing temperatures, we have chosen this procedure so as keep our silver island film preparations consistent with all our other reports of metal-enhanced fluorescence [119]. In these reports, the silver nanostructures were all fabricated under similar atmospheric conditions, where silver oxide layers are likely to have also readily formed [1-19].

In addition, the annealed surfaces produce larger fluorescence enhancements, an $\approx 50 \%$ increase as compared to films, which have not been thermally annealed. We rationale these increases in fluorescence of fluorophorecoated annealed films, as compared to the unannelaed films, in terms of the larger particles which are formed upon annealing, and their subsequent greater scattering cross sections and therefore greater ability to enhance fluorescence, as compared to smaller, unannealed $\mathrm{SiFs}$.

\section{MATERIALS AND METHODS}

\section{Materials}

Silver nitrate (99.9\%), sodium hydroxide ( $99.996 \%)$, ammonium hydroxide (30\%), trisodium citrate, D-glucose, and premium quality APS-coated glass slides $(75 \mathrm{~mm} \times 25 \mathrm{~mm})$ were obtained from SigmaAldrich. All chemicals were used as received.

\section{Formation of Silver Island Films (SiFs) on APS-Coated Glass Substrates}

In a typical $\mathrm{SiF}$ preparation, firstly, a solution of silver nitrate $(0.5 \mathrm{~g}$ in $60 \mathrm{~mL}$ of deionized water $)$ in a clean $100-\mathrm{mL}$ glass beaker equipped with a Teflon-coated stir bar is prepared and placed on a Corning stirring/hot plate. While stirring at the quickest speed, 8 drops $(\approx 200 \mu \mathrm{L})$ of freshly prepared $5 \%(\mathrm{w} / \mathrm{v})$ sodium hydroxide solution are added. This results in the formation of darkbrownish precipitates of silver particles. Approximately $2 \mathrm{~mL}$ of ammonium hydroxide is then added, drop by drop, to re-dissolve the precipitates. The clear solution is cooled to $5^{\circ} \mathrm{C}$ by placing the beaker in an ice bath, followed by soaking the APS-coated glass slides in the solution. While keeping the slides at $5^{\circ} \mathrm{C}$, a fresh solution of D-glucose $(0.72 \mathrm{~g}$ in $15 \mathrm{~mL}$ of water) is added. Subsequently, the temperature of the mixture is allowed to warm to $30^{\circ} \mathrm{C}$. As the color of the mixture turns from yellow green to yellowish brown, and the color of the slides become greenish, the slides are removed from the mixture, washed with water, and sonicated for $1 \mathrm{~min}$ at room temperature. The SiFs deposited slides were then rinsed with deionized water several times prior to the annealing and fluorescence experiments. This procedure typically produced films with an average island height of $\approx 50 \mathrm{~nm}$ (see Fig. 2 top) and with a $\approx 40 \%$ surface mass coverage [5].

\section{Synthesis of Silver Colloids and Preparation of Silver Colloid Films}

Two milliliters of $1.16 \mathrm{mM}$ trisodium citrate solution was added dropwise to a heated $\left(95^{\circ} \mathrm{C}\right)$ aqueous solution of $0.65 \mathrm{mM}$ of silver nitrate while stirring. The mixture was kept heated for $10 \mathrm{~min}$, and then it was cooled to room temperature.

Silver colloid films were prepared by immersing the APS-coated glass slides in silver colloid solution overnight. The silver colloid deposited slides were rinsed with deionized water several times prior to the annealing and fluorescence experiments.

\section{Annealing of Silver Island Films and Silver Colloid Films}

Annealing of silver island films and silver colloid films were performed by placing the silver-coated slides in a Thelco Laboratory Oven at various temperatures (75, 121,190 , and $250^{\circ} \mathrm{C}$ ) for up to $10 \mathrm{hr}$.

\section{Metal-Enhanced Fluorescence from Annealed Silver Island Films and Silver Colloids}

In previous reports of metal-enhanced fluorescence (MEF), our laboratories have coated silvered surfaces with fluorophore-labeled protein [1-3]. This experimental format has been adopted for two main reasons, the first, being that the protein coverage with Human Serum Albumin 


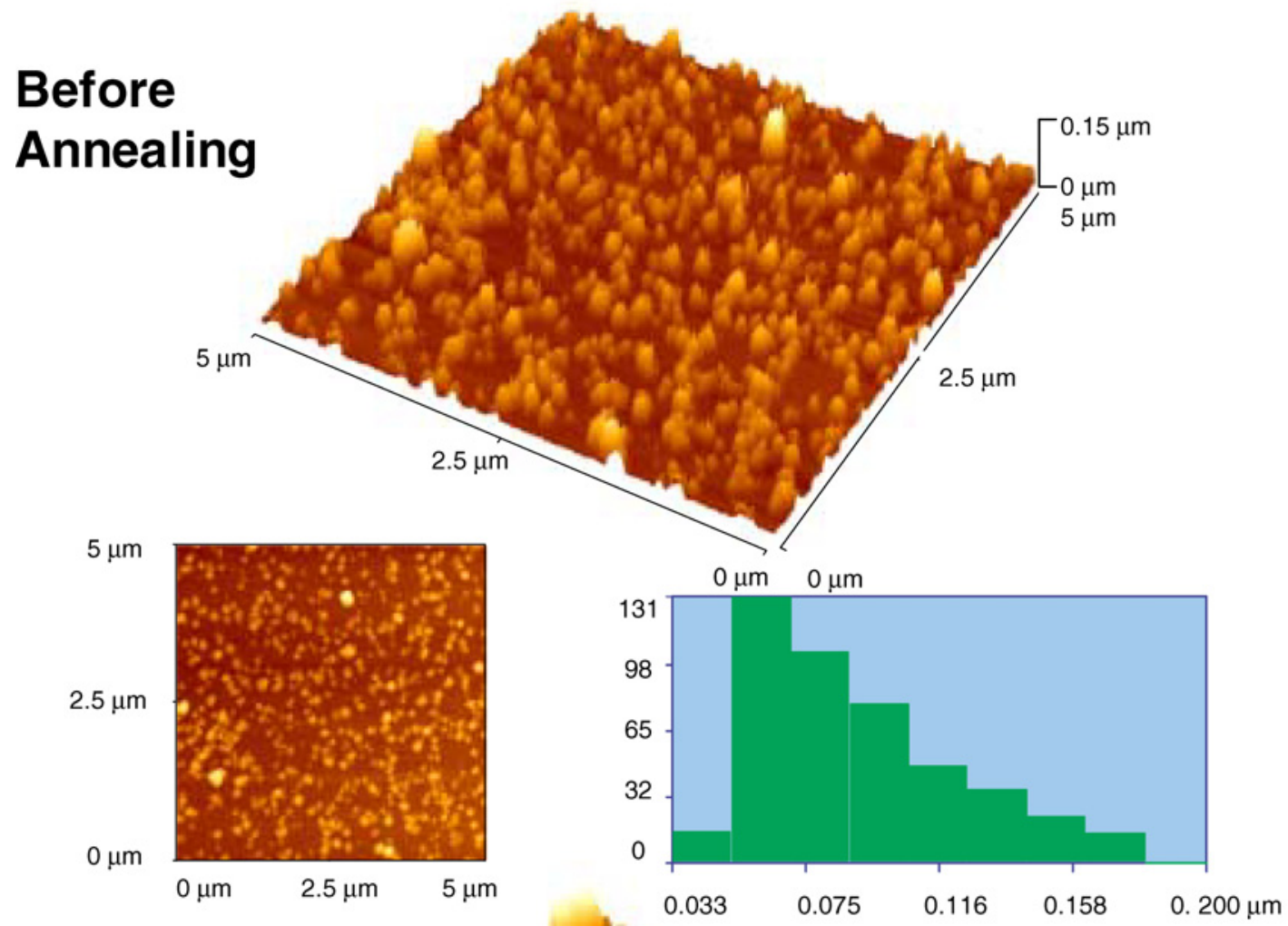

\section{Annealed SiFs, $75^{\circ} \mathrm{C}, 10 \mathrm{hr}$}

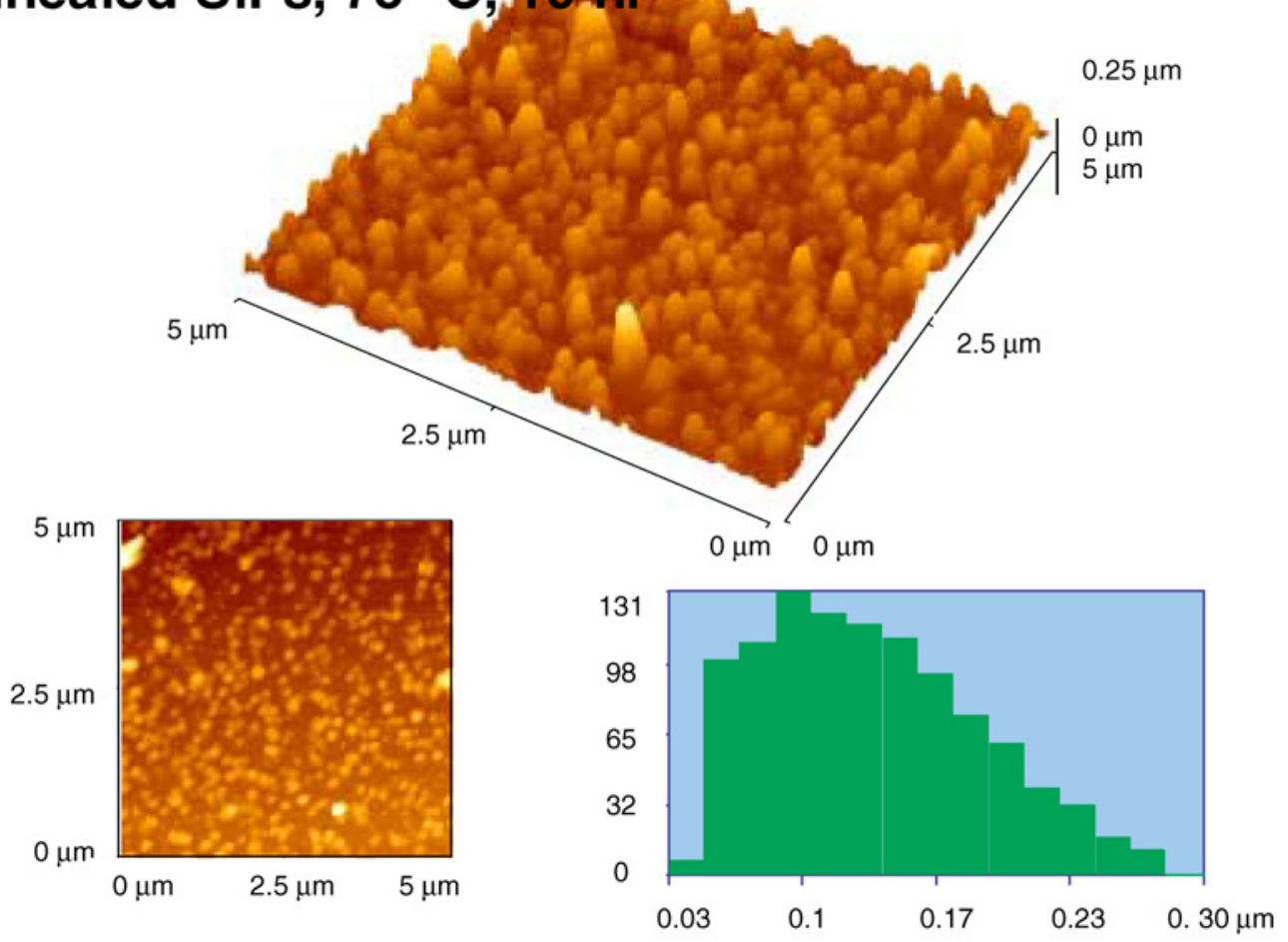

Fig. 2. AFM images of $\mathrm{SiFs}$ before (Top) and after thermal annealing at $75^{\circ} \mathrm{C}$ for $10 \mathrm{hr}$ (bottom). Histograms show surface height distributions. 
(HSA) is known to bind to silvered surfaces and indeed forms a monolayer $[1-3,11]$ and secondly, the dimensions of the protein being such that the protein allows for a mean $\approx 4 \mathrm{~nm}$ separation of the silver and the fluorophore, MEF being a through space phenomenon, as demonstrated by the late T. Cotton and indeed our laboratories [1-3]. In contrast, Surface-Enhanced Raman Scattering (SERS) is known to be a consequence of mostly contact between the species of interest and the silvered surface [1-4].

Binding the FITC-HSA to the silver island films and silver colloid films was accomplished by soaking in a $10 \mu \mathrm{M}$ FITC-HSA solution for $2 \mathrm{hr}$, followed by rinsing with water to remove the unbound material. Both the unsilvered and silvered films were coated with labeled HSA, which is known to passively absorb to noble metal surfaces and form $\mathrm{a} \approx 4 \mathrm{~nm}$ thick protein monolayer [1-3], allowing us to study the fluorescence spectral properties of noncovalent FITC-HSA complexes in the absence and presence of SiFs. By equally coating a silver film with FITC-HSA we were also able to determine the enhancement factor (benefit) obtained from using the silver, i.e., Intensity on Silver/Intensity on Unsilvered glass, given that both surfaces are known to have an $\approx$ equal monolayer coverage $[1-3,11]$.

\section{Characterization of Annealed Silver Island Films and Silver Colloid Films}

All absorption measurements were performed using a HP $8453 \mathrm{UV}$-Vis spectrophotometer. Fluorescence measurements on silver island films and silver colloid films were performed using a Varian Cary fluorescence spectrophotometer by placing the films on a stationary stage (positioned in front of the excitation source and the detector) equipped with mirrors to divert the excitation/emission to and from the sample.

AFM images were collected with an Atomic Force Microscope (TMX 2100 Explorer SPM, Veeco) equipped with an AFM dry scanner (the scanning area was $100 \mu \mathrm{m} \times 100 \mu \mathrm{m})$. Surfaces were imaged in air, in a tapping mode of operation, using SFM noncontact mode cantilevers (Veeco). The AFM scanner was calibrated using a standard calibration grid as well as by using gold nanoparticles, $100 \mathrm{~nm}$ in diameter from Ted Pella. Images were analyzed using SPMLab software.

Time-resolved intensity decays were measured using reverse start-stop time-correlated single-photon counting (TCSPC) [21] with a Becker and Hickl gmbh 630 SPC PC card and an unamplified MCP-PMT. Vertically polarized excitation at $\approx 440 \mathrm{~nm}$ was obtained using a pulsed laser diode, $1 \mathrm{MHz}$ repetition rate.

\section{RESULTS AND DISCUSSION}

Silver island films ( $\mathrm{SiFs}$ ), were annealed at various temperatures from 75 to $250^{\circ} \mathrm{C}$, Fig. 3. The silver plasmon absorption (extinction) spectra typically show a decrease in the $\approx 420 \mathrm{~nm}$ plasmon absorption band, as well as a shift to shorter wavelengths. This effect is more pronounced with increased temperature, where the spectral changes at $75^{\circ} \mathrm{C}$, are less dramatic than those at much higher annealing temperatures, c.f. Fig. 3, panels A and D. Subsequently by ratiometrically plotting the $420 \mathrm{~nm}$ absorption band as a function of annealing temperature and time, we can clearly see that the more significant changes to the silver islands are indeed occurring at higher temperatures, Fig. 4.

As well as measuring the plasmon absorption spectra we were able to get additional insights into the size and shape changes of the SiFs using Atomic Force Microscopy (AFM). Figure 2 shows a typical AFM image of a SiF both before and after annealing at $75^{\circ} \mathrm{C}$ for $10 \mathrm{hr}$, top and bottom, respectively. The right hand-side inserts show surface height histograms. Interestingly, while only slight changes in plasmon absorption were observed, Fig. 3, Panel A, the AFM image shows that much larger particles are present after annealing. In addition, by considering many spectra, taken with many samples under different conditions, we also observed the general trend that the spacing between the particles increased, and that the particles became spherical as a function of both time and annealing temperature. A height analysis of the surfaces revealed the structures to increase in size as a function of both annealing temperature and time, Fig. 5, where the changes in film height when annealed at $75^{\circ} \mathrm{C}$ are thought to be within the experimental error, i.e., no change in height is likely for a $75^{\circ} \mathrm{C}$ annealing temperature.

These changes in $\mathrm{SiF}$ morphology on surfaces have also been observed by others, [26-33] while developing platforms for Surface-Enhanced Raman Scattering [3133]. Similar to previous interpretations, our changes in plasmon extinction, coupled with an insight into the size and shape changes revealed by AFM, can be explained by net surface plasmon changes. While an increased particle size would be expected to result in a red shifted plasmon absorption maximum [34-36], the dominant influence here is the decreased interaction of neighboring $\mathrm{SiFs}$, as a result of an increased particle separation, noting that one would expect mass to be conserved on the surface during annealing. The interparticle dipole-dipole interactions are known to determine the width of the extinction bands of SiFs [37]. Hence, as the SiFs become spherical, larger, and more spaced, the net change in silver plasmon absorption is a blue shifted and decreased absorption 

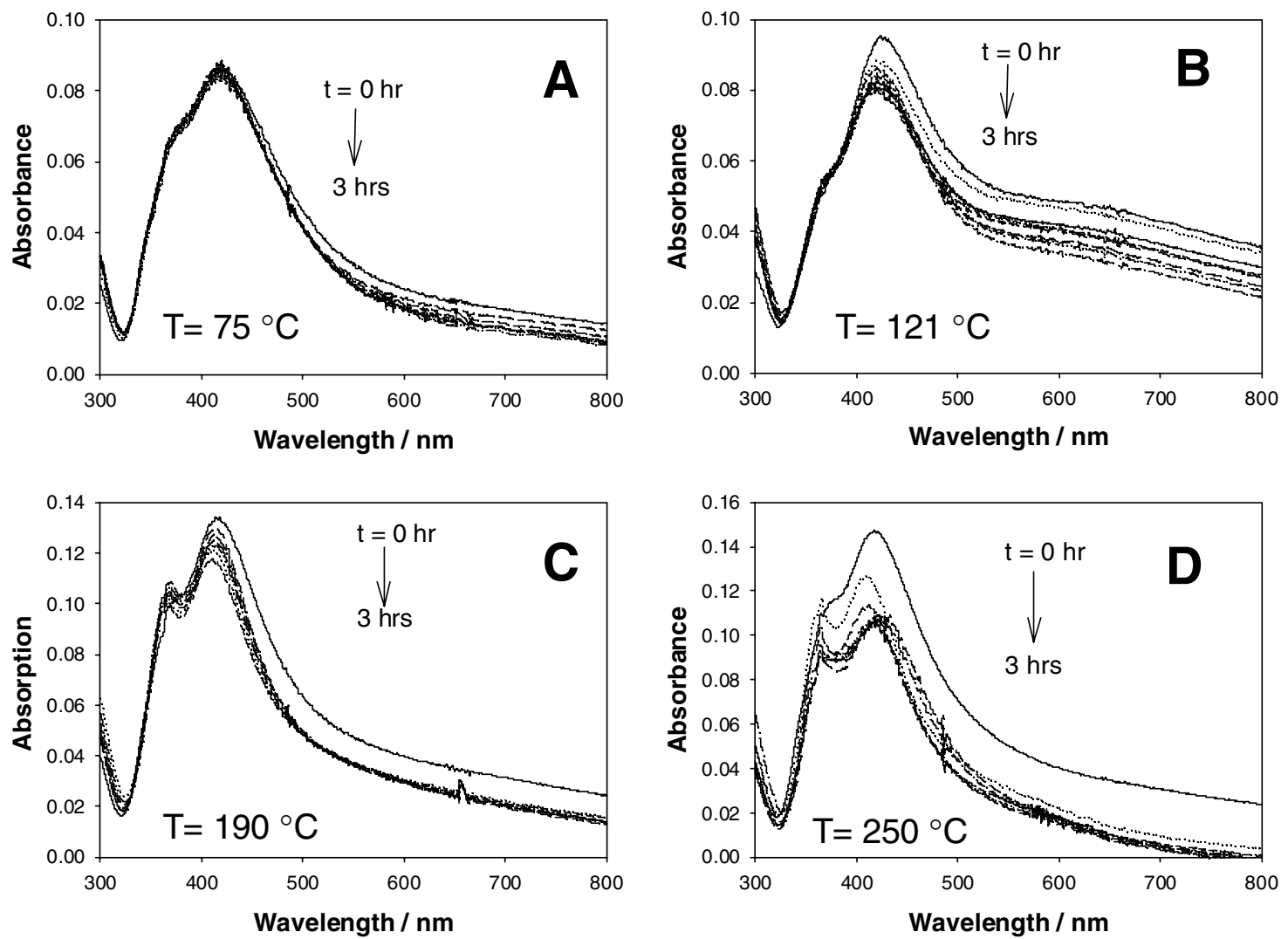

Fig. 3. Absorption spectra of $\mathrm{SiFs}$ after annealing at different temperatures. $\mathrm{A}-75^{\circ} \mathrm{C}, \mathrm{B}-121^{\circ} \mathrm{C}, \mathrm{C}-190^{\circ} \mathrm{C}$, and $\mathrm{D}-250^{\circ} \mathrm{C}$.

maximum. Interestingly for films annealed at $250^{\circ} \mathrm{C}$, then a slightly different behavior is observed, whereby the silver plasmon absorption eventually red shifts after initially shifting blue. This is thought to be due to the particles being sufficiently spaced that the dipole-dipole interactions become very weak, the plasmon absorption now dominated by the size and shape of the isolated particles.

The effects of thermally annealing silver colloid films were, however, markedly different. The plasmon absorption spectra both red shifted and increased for temperatures over $190^{\circ} \mathrm{C}$, Fig. 6. Interestingly, the size of the colloids did not significantly change from their initial 30-80 nm diameter size distribution, (data not shown) suggesting that the changes observed in the absorption spectra were dominated again by particle-particle dipole-dipole interactions [37], and not by size and shape.

To investigate the use of both annealed SiFs and colloidal films in metal-enhanced fluorescence, films were coated with fluorescein-labeled Human Serum Albumin (HSA), which passively absorbs onto the surface and is known to form a monolayer coverage [1-3]. Figure 7 top and middle shows that enhanced fluorescence can be observed from annealed SiFs and colloidal films, as compared to a control sample, which is a monolayer of labeled-protein-coated glass. By considering the enhancement factor, Fig. 7 bottom, which is the ratio of the

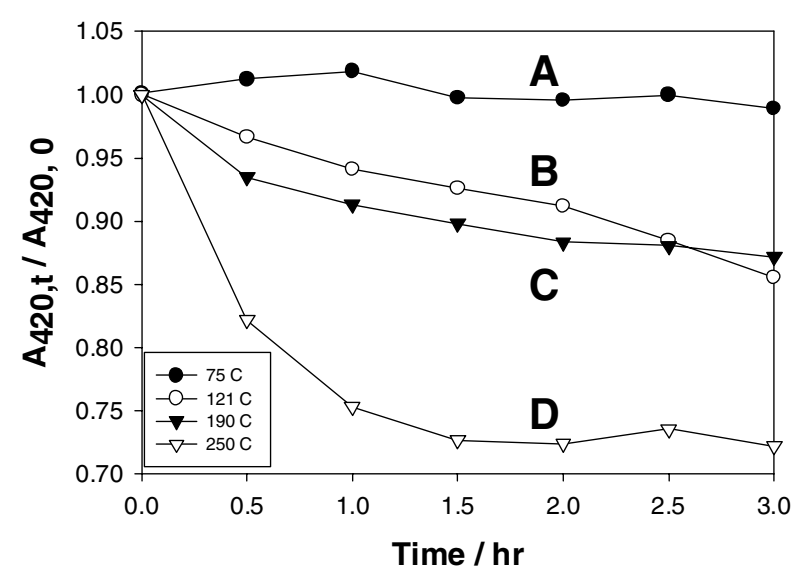

Fig. 4. Time-dependent change in absorption at $420 \mathrm{~nm}$ of SiFs after annealing at different temperatures. $\mathrm{A}-75^{\circ} \mathrm{C}, \mathrm{B}-121^{\circ} \mathrm{C}, \mathrm{C}-190^{\circ} \mathrm{C}$, and $\mathrm{D}-250^{\circ} \mathrm{C}$. 


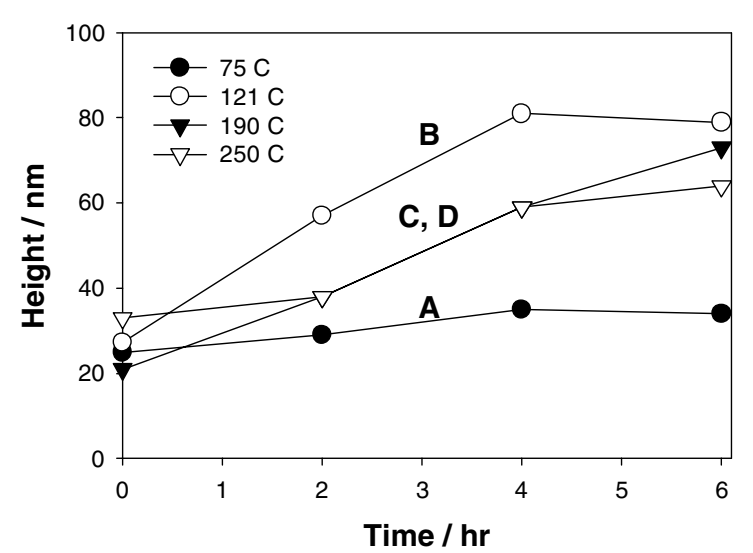

Fig. 5. Time-dependent change in height of SiFs after annealing at different temperatures. $\mathrm{A}-75^{\circ} \mathrm{C}, \mathrm{B}-121^{\circ} \mathrm{C}, \mathrm{C}-190^{\circ} \mathrm{C}$, and $\mathrm{D}-250^{\circ} \mathrm{C}$.

fluorescein emission from the annealed silver surfaces as compared to glass, we can see notable differences between annealed SiFs and colloids. Firstly, the enhanced fluorescence from colloidal films is generally larger than for SiFs. This finding is consistent with previous reports from our laboratory $[2,12]$ and is in part due to the enhanced electric fields around the colloids, which also can enhance fluorescence due to modifications in a fluorophores' excitation rate $[4,20]$. Secondly, the enhancements from colloidal films are approximately constant, irrespective of surface thermal annealing, which is in contrast to SiFs, which show an increase of up to $50 \%$ in the enhancement. This result suggests the use of preannealed silver island or colloid films as substrates in MEF, which involve modest temperature increases, such as in substrates for hybridization assays [7], or for use as reusable autoclavable clinical sensing platforms. In addition, Fig. 7 bottom shows sim-

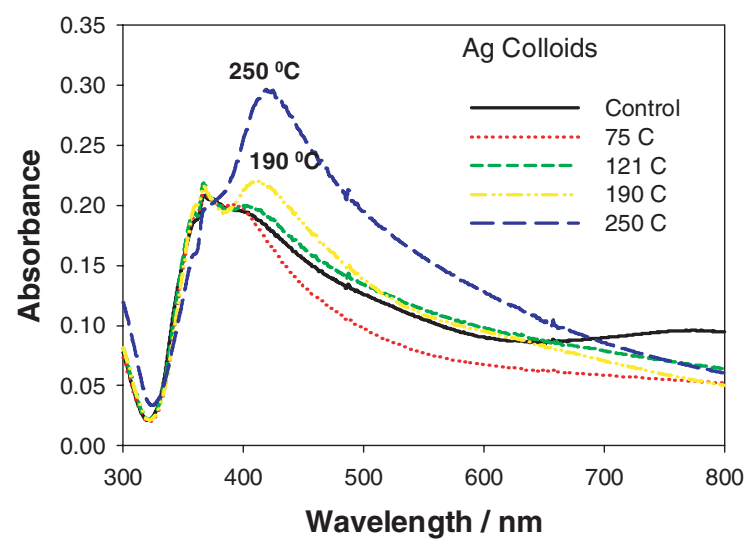

Fig. 6. Absorption spectra of silver colloids deposited on APS-coated glass slides after thermal annealing at different temperatures. ilar enhancements for $\mathrm{SiFs}$ and colloids after annealing at high temperature $\left(>190^{\circ} \mathrm{C}\right)$ for $3 \mathrm{hr}$. Given that both the plasmon absorption spectra and AFM images show a rearrangement from $\mathrm{SiFs}$ to colloids as a function of both temperature and annealing time, coupled with the fact that colloidal films undergo little shape and size change, then the similar fluorescence enhancements observed are considered to be due to there being similar nanostructures present on both the surfaces after annealing. While beyond the scope of our laboratory annealing equipment at this time, we question whether very similar surface structures would eventually be observed after annealing at higher temperatures than were employed in our study, and for longer periods of time? i.e., would the curves in Fig. 7 bottom eventually converge?

In many examples of fluorescence-based sensing it is fluorophore detectability that governs the utility and sensitivity of the sensing approach [21]. In general, the detectability of a fluorophore is determined by two factors: the extent of background emission from the sample and the photostability of the fluorophore. A highly photostable fluorophore, such as tetramethyl rhodamine [4] can undergo about $10^{6}$ excitation-relaxation cycles prior to photobleaching. While this can yield as many as $10^{4}$ detectable photons per fluorophore it should be realized that the vast majority of fluorophores photodegrade after far fewer excitation-emission event cycles [4,21]. Subsequently, we tested the photostability of fluoresceinHSA on the annealed SiFs as compared to an identical unannealed $\mathrm{SiF}$ sample as well as a glass control sample. Figure 8 top shows fluorescein-HSA emission as a function of time, excited at $470 \mathrm{~nm}$ and observed through a $500 \mathrm{~nm}$ long pass filter. The relative intensities of the plots reflect the benefits of using silvered surfaces for MEF, in that more detectable photons can be observed per unit time from the annealed $\mathrm{SiFs}$, as compared to both unannealed SiFs and indeed the glass control surface, where the integrated areas under the plots are proportional to the photon flux from the respective samples. By additionally adjusting the laser power to match the same initial steady-state intensities of the samples, Fig. 8 bottom, we can see that the annealed $\mathrm{SiF}$ produces more photostable fluorescein-HSA. This finding is consistent with a reduced fluorescein lifetime, the fluorescein in essence spending less time in an excited state, and therefore is less prone to photodestruction [1-3], i.e., it is more photostable.

We additionally measured the lifetime of the samples used in the photostability study, Fig. 9. The intensity decay shows that a much shorter lifetime is evident for fluorescein-HSA as compared to the glass control sample, further supporting our photostability observations. 

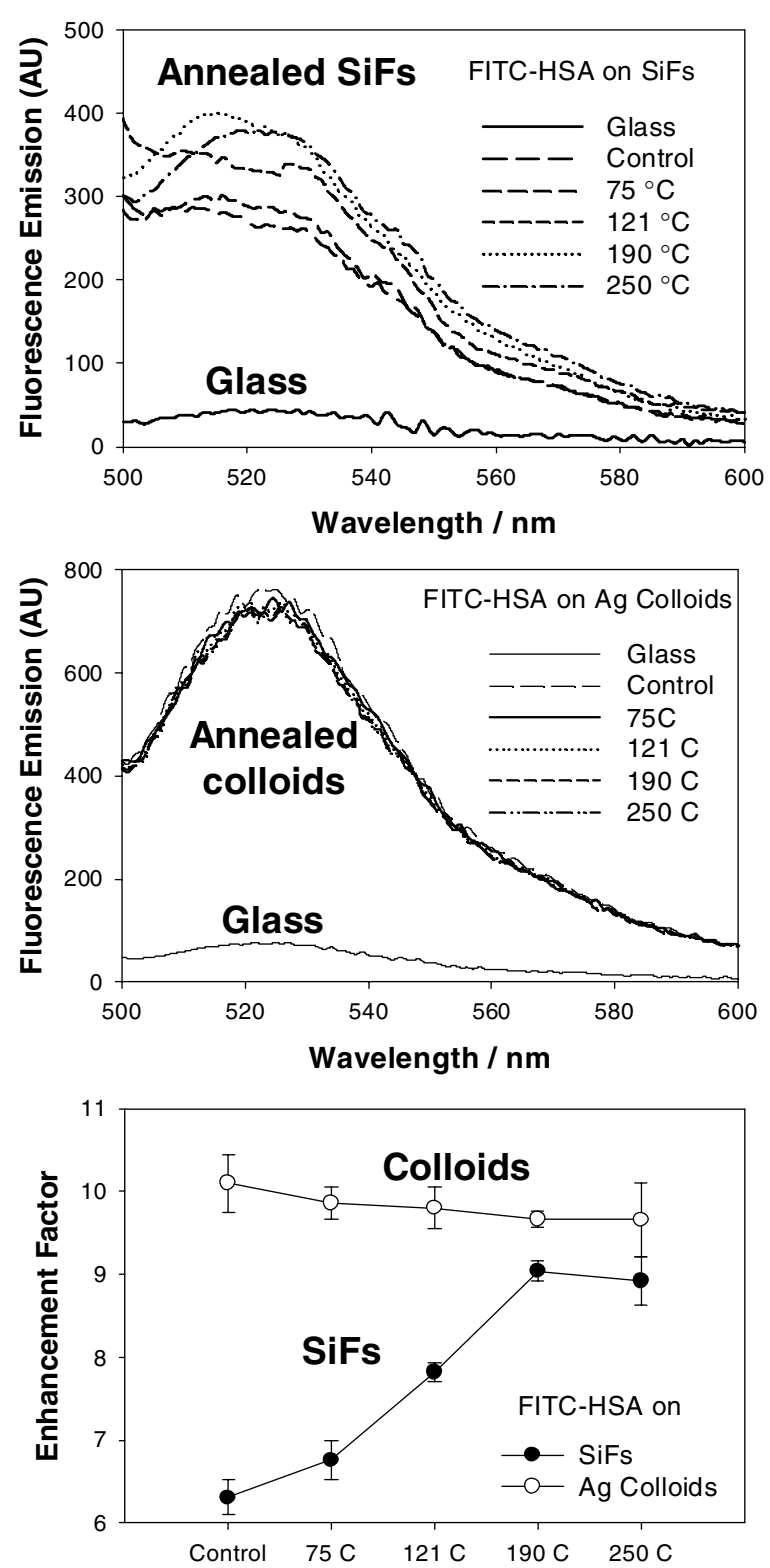

Fig. 7. Enhanced fluorescein-HSA emission on annealed SiFs (Top) and silver colloids (Middle), as compared to a glass control sample, which is unannealed. The annealing time was $3 \mathrm{hr}$. (Bottom) - The enhancement factor (mean of three measurements) as a function of annealing temperature, where the enhancement factor is the ratio of fluorescein emission on $\mathrm{SiFs}$ or colloids, as compared to unannealed fluorescein-HSA-coated glass.

Finally, it is interesting to note that the annealed films yield both increased emission from locally positioned fluorescein, as well as a reduced fluorescein lifetime. As briefly mentioned in the Introduction, this combination of an increased quantum yield, coupled with a decreased lifetime is unusual in fluorescence spectroscopy, and is
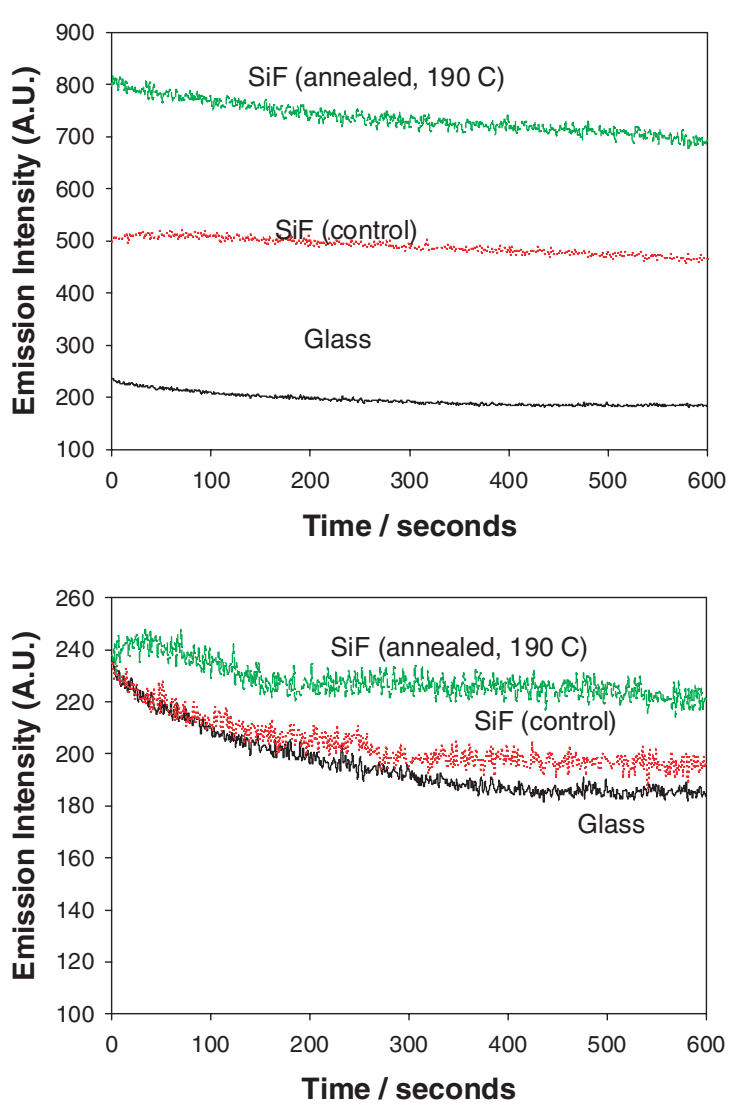

Fig. 8. Photostability of FITC on glass slides and SiFs before and after annealing at $190^{\circ} \mathrm{C}$. Top-Same laser power, Bottom-Laser power adjusted to give the same steady-state intensity.

consistent with a modification of the intrinsic radiative decay rate of the fluorophore, see Eqs. (1-4). This forms the basis of our earlier interpretations of metal-enhanced fluorescence $[1-5,20]$.

\section{Metal-Enhanced Fluorescence: Interpretation in Terms of Radiating Plasmons}

Until recently the emission of fluorophores in close proximity to metallic nanostructures was thought to originate solely from the fluorophore, the excited plasmons interacting with the fluorophore and changing its freespace spectral characteristics $[1-5,20]$. However, recently our interpretation of metal-enhanced fluorescence has changed somewhat [22] as shown in Fig. 1, to one whereby excited fluorophores can nonradiatively transfer energy to surface plasmons which in turn, radiate the fluorophores' photophysical characteristics, in essence the system radiates [22]. While there is very little experimental evidence to date that the metal-enhanced fluorescence phenomenon 


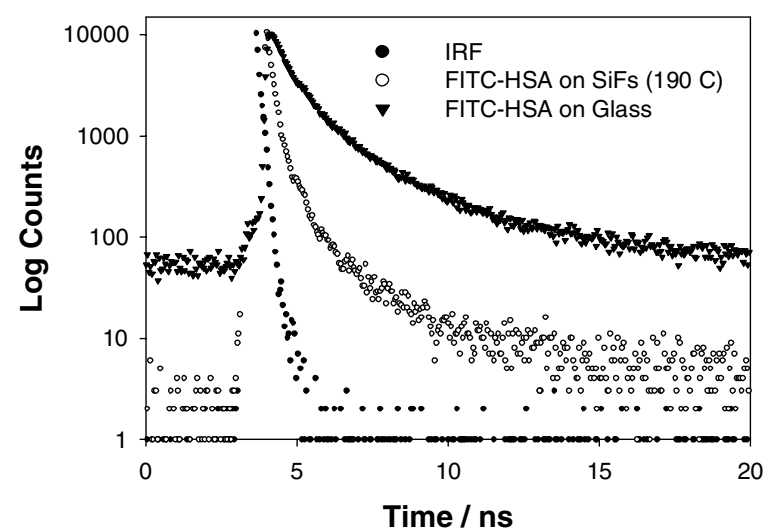

Fig. 9. Typical intensity decay of fluorescein-HSA-coated annealed $\operatorname{SiFs}\left(190^{\circ} \mathrm{C}\right)$ and glass. IRF-Instrumentation Response Function.

is due to fluorophore-coupled radiating plasmons, the data presented here for thermally annealed silver island films, certainly goes some ways to support this interpretation, as is described below.

It is known that surface plasmons can be created by illumination of thin continuous metal films under very unique optical conditions, such as through a prism, or a medium of high dielectric constant and with p-polarized light [22]. However, surface plasmons can also be created by direct illumination of metallic solution based colloids or nanostructures, or even by nanostructures bound to surfaces [22]. Illumination of nanostructures or colloids typically results in the visualization of strong colors, which is due to a combination of both absorption and scattering $[35,36]$. The term "absorption" is generically used for these nanostructures and colloids, but the correct term is "extinction," as there are both absorption and scattering components to the observed colors. Based on the recent radiating plasmon model postulated by our laboratories [22], small colloids are expected to quench fluorescence, because the absorption component of the extinction is dominant over scattering, while larger colloids or nanostructures are expected to result in enhanced fluorescence, as the scattering component is now dominant over the absorption component of the extinction spectra. Intuitively, by considering Eqs. (5) and (6) we expect the absorption term $C_{\mathrm{A}}$ to cause quenching, and the scattering term $C_{\mathrm{S}}$ to cause fluorescence enhancement due to fluorophore coupling (described in the next section in more detail). Examination of Eq. (5) shows that $C_{A}$ increases as the radius of the nanoparticle cubed, whereas $C_{S}$ increases as the radius to the sixth power. For this reason, larger nanostructures are expected to show greater fluorescence enhancements than smaller nanoparticles. While this understanding underpins Mie theory [35,36,38-40] for small spherical particles whose radius is less than $0.05 \lambda$, the general conditions and equations are still valid for much larger nanostructures, but considerably more complex [35,36,38-40].

With regard to the fluorescence enhancements observed here for annealed silver island films, it is thought that the fluorescence enhancement increase, Fig. 7 bottom, is due to the increased size of the nanostructures after annealing, the $C_{S}$ component of the extinction becoming more dominant than the absorption, $C_{A}$, component. Similarly, the annealed colloidal film data also bears out this interpretation, as the size of the surface-bound colloids remained approximately the same after annealing, the Fluorescein-HSA enhancement approximately constant as seen in Fig. 7 bottom. In addition, while the electric field effect or sometimes called the "lightening rod effect" $[4,20]$ is known to modify fluorophore absorption cross sections, thereby increasing the excitation rate of fluorophores, this effect is dependent on the close locality of the fluorophore to the nanostructures, and would not be expected to increase with an increase in interparticle spacing, as is observed here. Moreover, a modification in the excitation rate of fluorophores would not alter a fluorophores' fluorescence lifetime $[4,20]$. To the best of our knowledge this is the first experimental observation that supports the hypothesis of fluorophore-plasmon coupled emission from noncontinuous particulate films.

\section{An Increased Quantum Yield, Decreased Lifetime, and Plasmon-Coupled Emission}

While we have rationaled the observations of enhanced fluorescence intensities as a function of $\mathrm{SiF}$ annealing in terms of the increased $C_{\mathrm{S}}$ component of the extinction spectra, it is informative to comment on the quantum yield and lifetime changes observed, given that current thinking has slightly shifted from a radiative rate modification, as originally depicted by Eqs. (1-4) [1-3].

The lifetime of surface plasmons are known to be very short, on the order of tens of femtoseconds $[41,42]$. This suggests that in our radiating plasmon interpretation, the energy transfer is essentially one way, from fluorophore to metal [22]. The increased quantum yield of fluorophores in close proximity to metallic nanostructures can be understood as the result of rapid energy transfer to the plasmons, which then radiate to the far-field [22]. When discussing excited fluorophores near to metals, we assume that the near field is present while the fluorophore is in an excited state, i.e., a field around an oscillating dipole at distances closer than the wavelength. For far field radiation, we refer to a wave propagating away from its source, whether fluorophore or metal. For a fluorophore, 
the far-field wave exists after it releases a photon and returns to the ground state.

The concept of donor-acceptor emission was first described by Forster for donor-acceptor pairs [21]. However, our laboratories have recently shown experimentally for fluorophores that rapid energy transfer from a donor to an acceptor resulted in an overall increase in the quantum yield of the system, when the quantum yield of the acceptor is greater than the donor [43,44]. This effect occurs because the rate of Forster energy transfer is proportional to the radiative decay rate of the donor and is independent of the nonradiative decay rates. If the transfer rate is high, which is thought to be the case for fluorophoremetal combinations, then the energy is transferred before the donor can decay by the nonradiative pathways, which are inherently the same in the absence and presence of the acceptor.

The effect of transfer to the metal can be described well by the following equations, where the rate of energy transfer to plasmons, or groups of plasmons is provided for by Forster's theory, and is given by:

$$
k_{\mathrm{T}}=\frac{1}{\tau_{0}}\left(\frac{R_{0}}{r}\right)^{6}
$$

where $n=4$ or 3 for a plane or filled half-space of acceptors, respectively, $R_{0}$ is the well-known Forster distance, $r$ is the distance from the fluorophore to the acceptor (metal), and $\tau_{0}$ is the lifetime of the donor fluorophores in the absence of acceptors. Recalling the quantum yield and lifetime equations in the absence of metal, Eqs. (3) and (4), the efficiency of energy transfer into the plasmons is given by:

$$
E=\frac{k_{\mathrm{T}}}{\Gamma+k_{\mathrm{nr}}+k_{\mathrm{T}}}
$$

As the rate of transfer becomes larger than the inverse lifetime, then the transfer efficiency approaches unity.

The total emission of the fluorophore-metal combination can now be given by:

$$
I_{\mathrm{T}}=I_{\mathrm{F}}+I_{\mathrm{P}}=k \varepsilon_{\mathrm{F}} Q_{0}(1-E)+k \varepsilon_{\mathrm{F}} Q_{\mathrm{S}}
$$

where $I_{\mathrm{F}}$ is the fluorophore emission intensity and $I_{\mathrm{P}}$ is the emission due to the radiating plasmons, $Q_{\mathrm{S}}$ is the scattering quantum yield, $\varepsilon_{\mathrm{F}}$ is the absorption coefficient of the fluorophore, and $k$ is an instrumental constant describing collection efficiencies, etc. From Eq. (9) we can see that as the transfer efficiency, $E$, approaches $100 \%$ (unity), the total intensity becomes:

$$
I_{\mathrm{F}}=k \varepsilon_{\mathrm{F}} Q_{\mathrm{S}}
$$

This means that for fluorophore-metal combinations, the effective quantum yield of the fluorophore approaches unity and the overall quantum yield of the system becomes the quantum yield of scattering. Remarkably, this occurs irrespective of whether the fluorophore has a low or high quantum yield. This forms the basis of our recent radiating plasmon model [22].

In terms of the annealed $\mathrm{SiF}$ data presented in this paper, we see an overall increase in the fluorescence intensity as the size of the nanostructures increases, because the quantum yield of scattering is higher for larger structures than for smaller structures, i.e., competition between absorption (quenching) and scattering, the $C_{A}=$ radius $^{3}$ vs. $C_{S}=$ radius $^{6}$, c.f. Eqs. (5) and (6) $[35,36]$. The lifetime of fluorescein in the presence of metal also drops, similar to any donor in the presence of an efficient acceptor as depicted by Forster's theory [21].

\section{CONCLUSIONS}

In this paper we have shown that silver island films can undergo both size and shape changes as a function of thermal annealing between 75 and $250^{\circ} \mathrm{C}$. In contrast, silver colloid films, were relatively unperturbed by thermal annealing. When coated with a fluorophore-labeled protein, then metal-enhanced fluorescein fluorescence can be observed from the surfaces, up to a 10-fold increase as compared to surfaces having no silver. These findings suggest the use of annealed silver island films as reusable autoclavable substrates for clinical assays, or for elevated temperature-based sensing, such as for use in metal-enhanced fluorescence hybridization assays.

We have also rationaled the increases in fluorescence observed, not in terms of a fluorophore radiative decay rate modification as was previously postulated by our laboratories, but one which involves efficient energy transfer to surface plasmons, which then radiate, depending on the quantum efficiency of the scattering component of the particles' extinction spectrum, which is itself dependent on nanoparticle size. While the data presented here is not definitive proof of our new interpretation of metal-enhanced fluorescence, the size dependence of the silver nanostructures on fluorescence enhancement, certainly supports our interpretation and the new radiating plasmon model.

Finally, metal-enhanced fluorescence is a new and emerging tool in biotechnology. At present it is difficult to predict whether a particular sized metal structure will quench or even enhance fluorescence. Subsequently, more detailed studies of the sizes and shapes of nanostructures, and the locality of fluorophores are needed with regard to quantitative MEF. In this regard, the radiating plasmon model provides the first framework for this interpretation. 
Further studies by our laboratories are currently underway.

\section{ACKNOWLEDGMENT}

This work was supported by the NIH GM070929 and RR008119. Partial salary support to CDG and JRL from UMBI is also acknowledged.

\section{REFERENCES}

1. K. Aslan, I. Gryczynski, J. Malicka, E. Matveeva. J. R. Lakowicz, and C. D. Geddes (2005). Metal-enhanced fluorescence: An emerging tool in biotechnology. Curr. Opin. Biotechnol. 16(1), 55-62.

2. C. D. Geddes, K. Aslan, I. Gryczynski, J. Malicka, and J. R. Lakowicz (2004). in Noble Metal Nanostructure for MetalEnhanced Fluorescence [Review Chapter for Annual Reviews in Fluorescence 2004 ed.], Kluwer Academic/Plenum, New York, pp. $365-401$.

3. C. D. Geddes, K. Aslan, I. Gryczynski, J. Malicka, and J. R. Lakowicz (2005). in C. D. Geddes and J. R. Lakowicz (Eds.), Topics in Fluorescence in Fluorescence Spectroscopy [Radiative Decay Engineering], Kluwer Academic/Plenum, New York, pp. 405-448.

4. J. R. Lakowicz (2001). Radiative decay engineering: Biophysical and biomedical applications. Anal. Biochem. 298, 1-24.

5. J. R. Lakowicz, Y. Shen, S. D'Auria, J. Malicka, J. Fang, Z. Grcyzynski, and I. Gryczynski (2002). Radiative decay engineering 2. Effects of silver island films on fluorescence intensity, lifetimes, and resonance energy transfer. Anal. Biochem. 301, 261-277.

6. J. R. Lakowicz, Y. Shen, Z. Grcyzynski, S. D'Auria, and I. Gryczynski (2001). Intrinsic fluorescence from DNA can be enhanced by metallic particles. Biochem. Biophys. Res. Commun. 286, 875-879.

7. J. Malicka, I. Gryczynski, and J. R. Lakowicz (2003). DNA hybridization assays using metal-enhanced fluorescence. Biochem. Biophys. Res. Commun. 306, 213-218.

8. J. R. Lakowicz, J. Malicka, S. D'Auria, and I. Gryczynski (2003). Release of the self-quenching of fluorescence near silver metallic surfaces. Anal. Biochem. 320, 13-20.

9. K. Aslan, J. R. Lakowicz, H. Szmacinski, and C. D. Geddes (2005). Enhanced ratiometric $\mathrm{pH}$ sensing using SNAFL-2 on silver island films: Metal-enhanced fluorescence sensing. J. Fluoresc. 15(1), 3740.

10. E. Matveeva, Z. Grcyzynski, I. Gryczynski, J. Malicka, and J. R. Lakowicz (2004). Myoglobin immunoassay utilizing directional surface plasmon-coupled emission. Anal. Chem. 76(21), 6287-6292.

11. J. Malicka, I. Gryczynski, C. D. Geddes, and J. R. Lakowicz (2003). Metal-enhanced emission from indocyanince green: A new approach to in vivo imaging. J. Biomed. Opt. 8(3), 472-478.

12. C. D. Geddes, H. Cao, I. Gryczynski, Z. Grcyzynski, J. Fang, and J. R. Lakowicz (2003). Metal-enhanced fluorescence due to silver colloids on a planar surface: Potential applications of Indocyanine green to in vivo imaging. J. Phys. Chem. A 107(18), 3443-3449.

13. K. Aslan, J. R. Lakowicz, and C. D. Geddes (2005). Rapid deposition of triangular silver nanoplates on planar surfaces: Application to metal-enhanced fluorescence. J. Phys. Chem. B 107(13), 6247-6251.

14. K. Aslan, Z. Leonenko, J. R. Lakowicz, and C. D. Geddes (2005). Fast and slow deposition of silver nanorods on planar surfaces: Application to metal-enhanced fluorescence. J. Phys. Chem. B 109(8), 3157.

15. A. Parfenov, I. Gryczynski, J. Malicka, C. D. Geddes, and J. R. Lakowicz (2003). Enhanced fluorescence from fluorophores on fractal silver surfaces. J. Phys. Chem. B 107(34), 8829-8833.

16. C. D. Geddes, A. Parfenov, and J. R. Lakowicz (2003). Photodeposition of silver can result in metal-enhanced fluorescence. Appl. Spectrosc. 57(5), 526-531.
17. C. D. Geddes, A. Parfenov, D. Roll, J. Fang, and J. R. Lakowicz (2003). Electrochemical and laser deposition of silver for use in metal-enhanced fluorescence. Langmuir 19(15), 62366241.

18. K. Aslan, R. Badugu, J. R. Lakowicz, and C. D. Geddes (2005). Metal-enhanced fluorescence from plastic substrates. J. Fluoresc. 15(2), 99-104.

19. C. D. Geddes, A. Parfenov, D. Roll, I. Gryczynski, J. Malicka, and J. R. Lakowicz (2004). Roughened silver electrodes for use in metalenhanced fluorescence. Spectrochim. Acta Part A 60(8-9), 19771983.

20. C. D. Geddes and J. R. Lakowicz (2002). Metal-enhanced fluorescence. J. Fluoresc. 12(2), 121-129.

21. J. R. Lakowicz (1999). Principles of Fluorescence Spectroscopy, Kluwer, New York.

22. J. R. Lakowicz (2005). Radiative decay engineering 5: Metalenhanced fluorescence and plasmon emission. Anal. Biochem. 337(2), 171-194.

23. J. R. Lakowicz (2004). Radiative decay engineering 3: Surface plasmon-coupled directional emission. Anal. Biochem. 324(2), 153169.

24. I. Gryczynski, J. Malicka, Z. Gryczynski, and J. R. Lakowicz (2004). Radiative decay engineering 4. Experimental studies of surface plasmon-coupled directional emission. Anal. Biochem. 324(2), 170182.

25. C. D. Geddes, I. Gryczynski, J. Malicka, and J. R. Lakowicz (2004, February). Photonics Spectra.

26. C. E. Reed, J. Giergiel, S. Ushioda, and J. C. Hemminger (1985). Effects of annealing on the attenuated-total-reflection spectra of coldevaporated silver films. Phys. Rev. B 31(4), 1873-1880.

27. P. Royer, J. P. Goudonnet, R. J. Warmack, and T. L. Ferrell (1987). Substrate effects on surface-plasmon spectra in metal-island films, Phys. Rev. B 35(8), 3753-3759.

28. G. Andreasen, P. L. Schilardi, O. Azzaroni, and R. C. Salvarezza (2002). Thermal annealing of patterned metal surfaces. Langmuir 18, 10430-10434.

29. H. Bi, W. Cai, L. Zhang, D. Martin, and F. Träger (2002). Annealinginduced reversible change in optical absorption of Ag nanoparticles. Appl. Phys. Lett. 81(27), 5222-5224.

30. I. Doron-Mor, Z. Barkay, N. Filip-Granit, A. Vaskevich, and I. Rubinstein (2002). Ultrathin gold island films on silanized glass. Morphology and optical properties. Chem. Mater. 16(18), 34763483.

31. Y. Saito, J. J. Wang, D. A. Smith, and D. N. Batchelder (2002). A simple chemical method for the preparation of silver surfaces for efficient SERS. Langmuir 18(8), 2959-2961.

32. D. J. Semin and K. L. Rowlen (1994). Influence of vapor deposition parameters on SERS active Ag film morphology and optical properties. Anal. Chem. 66(23), 4324-4331.

33. S. E. Roark, D. J. Semin, and K. L. Rowlen (1996). Quantitative evaluation of SERS-active Ag film nanostructure by atomic force microscopy. Anal. Chem. 68(3), 473-480.

34. E. Hutter and J. H. Fendler (2004). Adv. Mater. 16(19), 1685.

35. J. Yguerabide and E. Yguerabide (1998). Light-scattering submicroscopic particles as highly fluorescent analogs and their use as tracer labels in clinical and biological applications: I. Theory. Anal. Biochem. 262, 137-156.

36. J. Yguerabide and E. Yguerabide (1998). Light-scattering submicroscopic particles as highly fluorescent analogs and their use as tracer labels in clinical and biological applications: II. Experimental characterization. Anal. Biochem. 262, 157176.

37. A. Feofanov, A. Ianoui, E. Kryukov, S. Maskevich, G. Vasiliuk, L. Kivach, and I. Nabiev (1997). Nondisturbing and stable SERS-active substrates with increased contribution of long-range component of Raman enhancement created by high-temperature annealing of thick metal films. Anal. Chem. 69(18), 37313740 . 
38. U. Kreibig and M. Vollmer (1995). Optical Properties of Metal Clusters, Springer, Berlin, p. 532.

39. D. L. Feldheim and C. A. Foss (2002). Synthesis, Characterization, and Applications [Metal Nanoparticles], Marcel Dekker, New York, p. 338.

40. C. G. Blatchford and M. Kerker (1982). Elastic scattering, absorption, and surface-enhanced Raman scattering by concentric spheres comprised of a metallic and a dielectric region. Phys. Rev. B 26(8), 4052-4063.

41. A. Wei (2004). Plasmonic Nanomaterials, Kluwer Academic/Plenum, New York, pp. 173-200.
42. M. Scharte, R. Porath, T. Ohms, A. Eschlimann, J. R. Krenn, H. Ditlbacher, F. R. Aussenegg, and A. Liebsch (2001). Do Mie plasmons have a longer lifetime on resonance than off resonance? Appl. Phys. B 73, 305-310.

43. B. P. Maliwal, Z. Gryczynski, and J. R. Lakowicz (2001). Longwavelength long-lifetime luminophores. Anal. Chem. 73, 42774285 .

44. J. S. Kang, G. Piszczek, and J. R. Lakowicz (2002). Enhanced emission induced by FRET from a long-lifetime, low quantum yield donor to a long-wavelength, high quantum yield acceptor. J. Fluoresc. 12(1), 97-103. 